\title{
Lidil
}

Revue de linguistique et de didactique des langues

$42 \mid 2010$

Multimodalité de la communication chez l'enfant

\section{Structuration physiologique de la gestuelle : modèle et tests}

Dominique Boutet

\section{(2) OpenEdition}

\section{Journals}

Édition électronique

URL : http://journals.openedition.org/lidil/3070

DOI : 10.4000/lidil.3070

ISSN : $1960-6052$

Éditeur

UGA Éditions/Université Grenoble Alpes

\section{Édition imprimée}

Date de publication : 30 novembre 2010

Pagination : 77-96

ISBN : 978-2-84310-184-7

ISSN : $1146-6480$

\section{Référence électronique}

Dominique Boutet, «Structuration physiologique de la gestuelle : modèle et tests », Lidil [En ligne], 42 | 2010, mis en ligne le 31 mai 2012, consulté le 01 mai 2019. URL : http://journals.openedition.org/ lidil/3070 ; DOI : 10.4000/lidil.3070 


\title{
Structuration physiologique de la gestuelle : modèle et tests
}

\author{
Dominique Boutet*
}

\begin{abstract}
RÉSUMÉ
La gestuelle coverbale est trop souvent associée à (contaminée par?) la parole co-occurrente. Elle relève pourtant d'une modalité non linéaire. La compositionnalité possible de la gestuelle ouvre sur une voie d'expression multilinéaire du sens. En dehors des emblèmes, aucun inventaire lexical ne permet d'étudier les productions gestuelles. Une approche physiologique répond à ce préalable : inventorier pour éclairer la composition. Après avoir présenté la structuration articulaire de la gestualité, les résultats d'un test d'assignation d'étiquettes montrent la validité d'une catégorisation structurée par les degrés de liberté (ddl) de la physiologie articulaire. Des proximités formelles sont explorées, elles forment une typologie qu'il reste à informer. En outre, des relations formelles et sémantiques émergent entre ces ddl. Elles seront abordées ici.
\end{abstract}

\section{ABSTRACT}

Coverbal gesture is too often associated with the cooccurrent speech. It comes under a non linear modality. The potential compositionality of gesture opens the way to a multilinear meaning. Except emblems, none lexical inventory allows studying the production of gestures. A physiological approach fits this preliminary: making an inventory to enlighten the compositionality. After the presentation of the articular structure of gestuality, the results of a test of assignment of tag reveal the validity of a categorization by physiological structured degrees of freedom. Some formal proximities are explored, they design a typology we have to inform. Furthermore formal and semantic connections emerge between these degrees of freedom. We tackle them here.

* Structures formelles du langage, UMR 7023/CNRS, Université Paris 8, Université d'Évry-Val d'Essonne. 
Le continuum de Kendon (1988) repris et largement diffusé par David McNeill correspond désormais à un des canons des études sur la gestualité. Sans entrer dans le détail de cette taxonomie, on peut dire qu'elle linéarise les phénomènes gestuels. Ils vont du moins conventionnel au plus linguistique selon quatre axes majeurs qui distribuent les instances suivantes :

Gesticulation $>$ Gestuelle $>$ Pantomime $>$ Emblème $>$ Langue des signes

En creux des quatre continua, le découpage en instances apparemment non hiérarchisées répond à un ordre souvent en présence de la gestualité, celui de la parole articulée en langage. Rien ne dit que cette ontologie vocale/verbale s'applique de la même manière à des phénomènes gestuels dont les contextes d'apparition divergent tant (pantomime, langue des signes, gesticulation). Nous voulons montrer que la physiologique articulaire structure des Unités gestuelles (UG désormais) qui constituent une base stable et indépendante de la typologie proposée dans le continuum, et qui sont susceptibles d'être investies par des significations.

Nous voulons montrer ici qu'une structuration corporelle opère pour certaines unités gestuelles. Ainsi, on ne saurait considérer le corps comme un simple support pouvant recevoir un ordre de constitution supérieur à lui, celui forgé par une linguistique durablement marquée par les langues vocales étudiées. Le corps ne porte pas seulement la gestualité, il l'informe. Plus qu'un support, c'est un substrat (Boutet et Cuxac, 2008).

L'approche présentée ici inverse la direction générale du continuum. Il s'agit de considérer des gestes iconiques, voire certains bâtons, comme des gestes emblématiques esquissés soumis à un tempo parolier d'une autre nature. De ce fait les gestes considérés, iconiques ou bâtons, pourraient être porteurs d'un sens esquissé comme autant de gestes non aboutis. Une primauté du sens est donnée aux gestes au détriment - on va le voir - d'une appréhension visuelle de leurs formes. En revanche, des filiations formelles de ces gestes basées sur une structuration physiologique fournissent le substrat sur lequel vont se rassembler des unités pourtant redevables de différences visuelles importantes.

Nous proposons ici d'exposer dans une première partie la matérialité de la gestualité à travers le modèle physiologique de structuration des gestes. Pour l'essentiel, non seulement celui-ci favorise l'émergence de formes gestuelles stabilisées mais en plus il organise la signification 
de ces unités gestuelles par des composantes identifiables, les degrés de liberté insérés dans des schémas d'action. L'organisation proposée est testée à travers une tâche d'assignation d'étiquettes demandée selon une méthode des juges. La description de ces tests ainsi que leurs résultats sont exposés dans une deuxième partie.

\section{Présentation de l'approche physiologique}

Le membre supérieur est décomposable en segments. Il présente donc une infrastructure qui sous-tend l'ensemble des mouvements possibles : les degrés de liberté et leurs combinaisons (Kapandji, 1997). Répertoriés, ces degrés ne sont pas si indépendants qu'il parait au premier abord. D'ailleurs, ils n'ont pas tous une amplitude fixée; ils peuvent covarier. En outre, ils ne sont pas exempts de mouvements involontaires déterminés mécaniquement par la forme des segments et de leurs articulations (pour une présentation plus détaillée voir Boutet, 2008). Des mouvements induits affectent parfois à distance des segments non adjacents. Nous verrons également que les rapports géométriques des axes de rotation d'un même segment peuvent changer au cours d'un geste. Commençons la description par l'extrémité la plus libre : la main.

\section{La main}

Ce segment présente 3 degrés de liberté correspondant à trois axes de rotation perpendiculaires les uns aux autres en position de repos (i.e. paume ouverte vers l'avant, la main ballante le long de l'avant-bras, lui-même dans le prolongement du bras, ce dernier étant sur le côté du corps). La pronation et la supination ${ }^{1}$ composent les deux pôles d'un degré de liberté de $180^{\circ}$ d'amplitude dans à peu près toutes les positions de l'avant-bras à l'exception d'une position de flexion de $80^{\circ}$ pour l'avant-bras (main collée à l'épaule) pour laquelle la pronation ne dépasse pas $45^{\circ}$. Une bonne représentation du mouvement de ce degré de liberté correspond au geste associé à la comptine «Ainsi font font

1. La pronosupination est un degré de liberté de la main dont le mouvement de $180^{\circ}$ tourne autour d'un axe de rotation qui court le long du radius et du cubitus. Ainsi les points de rotation se situent à la fois au niveau du poignet et également du côté du coude à l'insertion des deux os de l'avant-bras. Ce degré de liberté se traduit par un mouvement de rotation de la main perpendiculaire à l'axe formé par le radius et le cubitus. 
font les petites marionnettes ». La flexion/extension ${ }^{2}$ de $180^{\circ}$ compose un autre degré de liberté; elle est d'une amplitude équivalente à celle de la pronosupination. Lorsque les doigts pointent en avant ainsi que l'avant-bras, le mouvement de flexion extension porte la paume vers soi (flexion) ou vers l'avant (extension). L'orientation des axes de rotation respectifs ne varie pas pendant un mouvement. Le troisième degré de liberté le plus atypique de la main, l'abduction/adduction ${ }^{3}$, est de faible amplitude $\left(35^{\circ}\right.$ du côté de l'adduction - lorsque dans le plan de la paume le mouvement de la main oriente celle-ci vers l'auriculaire-, et $15^{\circ}$ du côté opposé - abduction - du côté du pouce).

Cette amplitude restreinte amène bien plus rapidement vers un transfert du mouvement sur l'avant-bras que pour les deux autres degrés de liberté. Au demeurant, l'orientation de l'axe de rotation de l'abduction/adduction varie à mesure que la main se rapproche d'une flexion ou d'une extension maximale (Kapandji, 1997, p. 142). Cette variation peut rendre parallèle et finalement coïncidant cet axe de l'abduction/adduction avec celui qui régit les mouvements de pronosupination. Confondus, ces deux degrés de liberté varient de manière polaire et symétrique : la flexion et l'extension inversent la liaison des deux autres degrés de liberté. Par exemple, le geste oscillant qui signifie «Au revoir» pour lequel la main en extension totale lie deux choses: la pronation avec l'abduction (l'un ne peut être fait sans l'autre) pour le mouvement vers l'index, et à l'opposé, la supination avec l'adduction lorsque le mouvement oscille dans l'autre direction, vers l'auriculaire. Pour un autre geste également oscillant qui place la main dans une position de flexion maximale cette fois : paraphrasé «Holàlà» (pointe des doigts vers soi, main fléchie au maximum, voir Calbris, 1990, p. 2 et 4) le mouvement de pronation va de pair cette fois avec celui d'adduction

2. La flexion/extension manuelle tourne autour d'un axe de rotation qui traverse la poignet dans sa plus grande largeur. Le mouvement de ce degré de liberté perpendiculaire à cet axe rapproche la paume vers l'intérieur de l'avant-bras (flexion) et l'en éloigne jusqu'à la butée articulaire située à $90^{\circ}$ pour le mouvement d'extension.

3. L'abduction/adduction, degré de liberté de la main, est d'une amplitude réduite comparativement aux deux autres degrés de liberté. L'axe de rotation autour duquel la main tourne passe à travers le poignet, de sa face intérieure à sa face extérieure et plutôt en avant vers la paume. Ainsi le mouvement que cet axe de rotation permet amène la main à bouger dans la direction du pouce (abduction) et vers l'auriculaire (adduction). 
(du côté de l'auriculaire), tandis que le mouvement vers l'index (abduction) est coïncident de la supination. Bien évidemment cet alignement d'axes est une tendance qui augmente à mesure qu'on se rapproche d'une position de flexion/extension maximale. Ainsi, elle exerce son effet sur la quasi-totalité de l'amplitude de flexion/extension à l'exclusion de la partie neutre.

Par ailleurs, une association de mouvements de deux degrés de liberté exerce une force sur la main dont le moment a un impact sur le troisième degré de liberté : elle oriente vers un mouvement induit de l'un des deux pôles. Du geste «Vous filez tout droit» en passant par celui d' «Attention à la fessée» jusqu'à celui d' «Au revoir», la position d'extension de la main croit et la coïncidence entre la pronation et l'abduction (mouvement du côté du pouce) augmente. Ainsi, sur le principe qu'on vient de décrire, on a toujours un mouvement induit - involontaire - dont l'impact en termes de pôles sur le troisième degré de liberté dépend de la différence de forces exercées par les mouvements volontaires qui affectent les deux autres degrés de liberté, mais aussi du nombre de degrés restant à l'amplitude maximale et des variations géométriques des axes de rotation. L'ensemble de ces facteurs permet de dresser une liste et une représentation de schémas d'action simples (24 en tout) sous forme de mouvements volontaires et liés à gauche du signe «>» et de mouvement induit à droite (voir schéma 1).

\section{L'avant-bras}

L'avant-bras (voir schéma 2) présente 2 degrés de liberté (la flexion/ extension ${ }^{4}$ et la rotation intérieure/extérieure ${ }^{5}$ ) dont les amplitudes d'au moins $170^{\circ}$ ont la particularité de ne pas être visibles dans toutes les

4. La flexion/extension de l'avant-bras amène celui-ci vers l'intérieur du bras ou l'en éloigne (l'extension $0^{\circ}$ équivaut à la position de l'avant-bras dans l'alignement du bras). Son amplitude est d'environ $170^{\circ}$ et l'axe de rotation autour duquel ce ddl bouge passe par le coude perpendiculairement au mouvement.

5. La rotation intérieure/extérieure de l'avant-bras est perpendiculaire à un axe qui court le long de l'humérus. Lorsque l'avant-bras est horizontal tandis que le bras est vertical et vers le bas, le mouvement de rotation intérieure/extérieure amène l'avant-bras à balayer horizontalement l'espace situé devant et sur les côtés. Lorsque l'avant-bras est dans l'alignement du bras et le long du corps, la rotation intérieure/extérieure est difficilement discernable d'un mouvement de pronosupination et s'ajoute à ce degré de liberté manuel. 
positions au moins pour la rotation. Ainsi, lorsque l'avant-bras forme un angle droit avec le bras (flexion de l'avant-bras de $90^{\circ}$ dirigé vers l'avant), la rotation intérieure/extérieure de l'avant-bras amène la pointe des doigts à tracer un demi-cercle dans un plan transversal au niveau de l'abdomen. À l'instar des longitudes sur un globe terrestre, lorsque la main se rapproche de l'épaule grâce à un mouvement de flexion de l'avant-bras, la distance séparant deux degrés de longitude diminue jusqu'à ce que l'ensemble de l'amplitude de la rotation intérieure/extérieure ne couvre plus qu'un arc de cercle de rayon très restreint pour une position de flexion totale. L'amplitude de la rotation intérieure/ extérieure couvre une distance bien plus importante à l'équateur - pour filer la métaphore du globe terrestre - qu'aux pôles. Ainsi par exemple, la vision, ne rendant compte que de la distance parcourue, interprète de manière distincte la même différence angulaire de rotation intérieure ici et là.

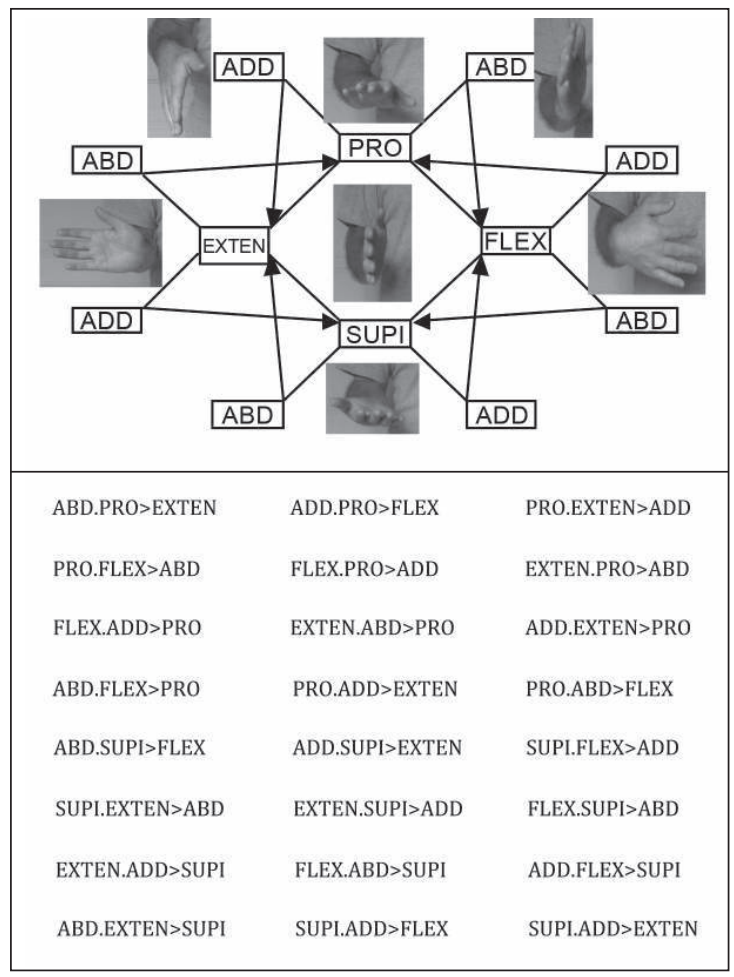

Schéma 1. - Figure des abouchements entre les degrés de liberté de la main. 
Dans l'autre direction, plus on se rapproche d'une extension totale de l'avant-bras (avant-bras dans le même alignement que le bras), plus le même phénomène exerce son effet, jusqu'à une particularité ajoutée, celle d'une coïncidence entre les axes de rotation de la rotation (intérieure/extérieure) de l'avant-bras et de la pronosupination de la main (l'avant bras, porteur de l'axe de rotation de la pronosupination est dans l'alignement du bras, porteur de l'axe de la rotation intérieure/extérieure). Ces deux mouvements, qui n'appartiennent pourtant pas au même segment, sont dès lors difficilement discernables. Ce qu'on en perçoit n'est rien d'autre qu'une rotation de la main sur elle-même, rotation qui ressemble fort à la pronosupination. Naturellement, ce phénomène que l'on vient de voir, de diminution de la distance parcourue, croit à mesure qu'on s'éloigne d'une position médiane de l'avant-bras, autour d'une flexion de $90^{\circ}$ (angle droit entre le bras et l'avant-bras), à l'instar de ce qui se passe pour la flexion/extension de la main. De plus l'espace délimité autant que créé par les deux degrés de liberté de l'avant-bras est semi-ovoïde. À ses limites, les degrés de liberté se confondent visuellement. Par exemple, la main droite posée sur le cœur tandis que le coude est collé au côté (flexion de l'avant-bras d'environ $60^{\circ}$ ) peut glisser progressivement vers l'épaule (celle porteuse du bras en mouvement) jusqu'à la toucher. Pour ce faire, un mouvement de flexion de l'avant-bras est suivi d'un mouvement de rotation extérieure sans que l'œil délimite exactement quand on passe de l'un à l'autre degré de liberté.

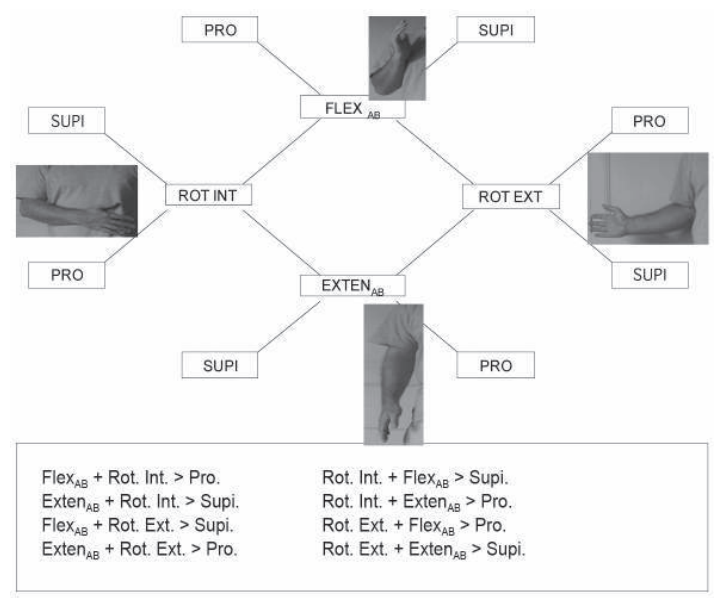

Schéma 2. - Figure des abouchements entre les degrés de liberté de l'avant-bras et la pronosupination. 
Quand un mouvement simultané affecte les deux degrés de l'avantbras, on a une répercussion involontaire sur la main pour la pronosupination. Ici cette répercussion induite quoique trans-segmentale est aussi polaire et symétrique. Voici ci-dessous une représentation des combinaisons sous forme schématique. On peut remarquer une sensibilité à la différence de forces exercées sur les deux degrés de liberté qui bougent de manière volontaire (figurés dans la liste sous forme d'ordres différents) et une accentuation de l'induction à mesure qu'on se rapproche de l'amplitude maximale de l'un des deux pôles en mouvement volontaire.

\section{Le bras}

Le bras, quant à lui, comporte deux degrés de liberté d'au moins $180^{\circ}$ d'amplitude chacun; l'un - l'abduction/adduction - amène un mouvement dans un plan frontal, d'une position basse où la main collée à la cuisse peut être projetée en haut, à l'aplomb de l'épaule en passant par le côté; l'autre degré de liberté - la flexion/extension - est effectuée dans un plan para-sagittal. Partant de la position de la main atteinte par une abduction de $180^{\circ}$, en haut et au-dessus de l'épaule, une extension d'au moins $180^{\circ}$ voit les segments du membre supérieur alignés passer par l'avant puis atteindre en bas la position initiale, voire remonter vers l'arrière. Là encore un phénomène d'induction de mouvement, transsegmental apparait et lie de manière symétrique et polaire un mouvement d'abduction (volontaire) à un mouvement de rotation intérieure et de pronation (involontaires), tandis que l'adduction du bras (sens horaire pour le gesteur) induit un mouvement de rotation extérieure et de supination involontaires (Kapandji, 1997, p. 22, pour une présentation du paradoxe de Codman et discussion).

\section{Les flux de propagation du mouvement}

Tous les segments vus sont liés entre eux par une structuration physiologique articulaire telle qu'à côté des transferts inertiels des moments des forces en présence existent des abouchements transitoires de degrés de liberté. Ceux-ci, à moins d'un mouvement volontaire du pôle inverse à celui du mouvement induit, fusionnent transitoirement avec le(s) degré(s) de liberté volontaire(s). Ce type de transferts de mouvement, structurellement inscrit, est plus rapide et plus constant que celui dû à l'inertie. Le liage involontaire entre pôles réserve une part belle à la pronosupination qui est concernée à chaque fois ; cependant dans 
un geste impliquant l'ensemble des segments du membre supérieur, le complexe de pôles en action, transférés involontairement ou non, peut amener à des non-congruences. Alors qu'un mouvement de pronation involontaire est attendu comme répercussion, le transfert inertiel d'un segment sur l'autre par exemple oriente vers une supination. On observe alors une compétition entre les deux pôles et finalement le mouvement qui en résulte.

Fixons un système gestuel composé d'inerties et des structurations décrites. Au sein de ce système, seuls deux degrés de liberté bougent volontairement ${ }^{6}$. Les mouvements sont transférés : le système distribue finalement un flux de propagation du mouvement soit proximal-distal (de l'épaule aux doigts) soit distal-proximal (propagation opposée). Les éléments qui participent à l'orientation générale du flux sont :

- les inerties de chaque segment qui donnent un gradient décroissant global par segment, du bras aux doigts ;

- les transferts structurels que nous avons vus;

- l'amplitude des degrés de liberté et particulièrement la distance restant à l'amplitude maximale ;

- et le différentiel des forces entre les deux degrés de liberté en mouvement volontaire.

\section{Émergence de schémas d'actions}

Sans entrer dans les détails de constitution des schémas d'actions, quarante unités gestuelles simples (UG, désormais) émergent à partir d'un double mouvement volontaire de deux degrés de liberté d'un segment ${ }^{7}$. Ce double mouvement volontaire sur un segment diffuse sur tout ou partie du membre supérieur. Il compose finalement un schéma d'action unique (ensemble de mouvements de degrés de liberté reliés entre eux) représentant la matrice d'une UG.

6. Il semble que nous pourrions nous placer dans un autre système dans lequel par exemple deux degrés de liberté du même segment bougeraient de manière volontaire avec en plus une position de départ très marquée de l'un des degrés de liberté en mouvement. L'ensemble des possibilités n'est pas encore exploré et reste un champ d'investigation encore très ouvert.

7. C'est donc sans compter les gestes complexes mettant en mouvement de manière volontaire trois degrés de liberté ou plus. De même, des compositions de mouvements et de positions bloquées doivent amener à l'émergence d'autres unités. 
Ces UG remplissent l'espace multidimensionnel de possibilités physiologiques stabilisées et différenciées du membre supérieur - à l'exception des combinaisons des doigts et du pouce. Il s'agit bien ici d'un espace non métrique pour lequel les distances n'ont en conséquence pas de réalité, il s'agit également d'un espace à plusieurs allocentrages (au moins autant que de segments), c'est-à-dire d'un espace non égocentré. Enfin, il s'agit d'un espace dont la constitution n'est pas visuelle. L'autoorganisation des UG est donc fondée sur des déterminations physiologiques et physiques de déploiement du mouvement sur des degrés de liberté (ddl). Les différentes réalisations de ces UG répondent à un autocontrôle proprioceptif. En fait, les réalisations que chaque UG déploie captent des enveloppes de formes. Celles-ci composent un réseau assez mêlé d'entre-croisements gestuels. Au point qu'il semble extrêmement long de pouvoir déterminer l'ensemble des variations d'amplitudes rassemblées sous une même UG, sans compter les transferts complets sur un autre segment (appelons-le support) que celui sur lequel l'unité gestuelle émerge et finalement se génère (ce sera le segment substrat). Ainsi, un geste organisé sur le bras, c'est-à-dire à partir de l'articulation de l'épaule, peut être partiellement transféré sur un segment adjacent comme l'avant-bras, il débordera de toute façon sur la main sous la forme d'un mouvement involontaire de pronosupination, comme nous l'avons vu. En respectant le schéma d'action de l'UG, le transfert du mouvement peut être total et finalement le geste peut être réalisé uniquement sur la main - et non pas par la main. La forme ainsi déployée sur la main capture une enveloppe plus qu'une forme unique (parce que dans un cas de transfert partiel, les variations d'amplitude de la même UG seront moindres), à côté de ce que la main génère en tant que substrat et non plus comme simple support. Ce qui est valable pour le bras l'est également pour les UG générées sur l'avant-bras qui débordent sur la main comme support cette fois encore.

Cette distinction substrat/support est indispensable pour comprendre la génération de gestes d'une part et la zone de variations des unités ainsi constituées, d'autre part. Rappelons qu'ici les variations gestuelles de chaque UG ne sont pas repérables selon un cadre de référence égocentré (un repère haut, bas, avant, arrière ne signifie rien), ni selon une modalité visuelle, mais qu'elles appartiennent en propre à la matérialité corporelle et à la filiation formelle qu'offre cette matérialité. Si la filiation de réalisations à une même unité gestuelle constituée selon des paramètres physiologiques et physiques peut toujours être déployée sur le support membre supérieur, elle peut également être réduite aux 
mouvements de quelques degrés de liberté sur un segment support éloigné de son centre d'organisation. Jusqu'où va la réduction formelle de ces filiations? En termes de distances segmentales, et en termes de nombre de degrés de liberté impliqués dans la réalisation? En somme, constatant l'émergence et la constitution d'unités gestuelles autour de schémas d'action comme autant d'emblèmes potentiels, jusqu'où va la réduction de ceux-ci en gestes plutôt redevables d'une potentialité d'interprétation en gestes dits iconiques dans la typologie de McNeill? Ces réductions du nombre de degrés de liberté en mouvement pour certaines réalisations permettent au locuteur/gesteur de s'adapter au débit parolier et la subsidiarité segmentale accroit les possibilités d'expression gestuelle par autant de segments disponibles hic et nunc.

\section{Tableau d'Unités gestuelles}

Nous ne présentons ici que 20 des 40 UG avec leur schéma d'actions respectif accompagné de leur proposition d'étiquette en guise d'unité de sens. Ces dernières relèvent de l'expérience et de l'intuition de l'auteur. La validité de cette assignation est testée (voir infra, partie 2). Le tableau 1 est organisé autour d'une césure verticale qui répartit de part et d'autre les inversions polaires des degrés de liberté manuels Flexion/Extension et Pronation/Supination. Une ligne horizontale sépare les UG en 2 blocs, 12 en haut, 8 en bas. Chacun de ces blocs est lui-même divisé par une ligne verticale. On a donc 4 sous-blocs en tout regroupés par pôles. Dans les deux sous-blocs supérieurs on distingue les UG 9, 10, 11 et 12 - les plus à l'extérieur - dont le substrat est l'avant-bras ou le bras. Sous l'étiquette de chaque geste figure le schéma d'actions organisé en ligne manuelle (de gauche à droite des quantités de mouvements décroissants), en ligne d'avant-bras (INT et EXT : abréviations de rotation intérieure et extérieure) et parfois en ligne du bras. Chaque UG est numérotée. La composante volontaire du segment substrat de chaque schéma d'action est soulignée. À position équivalente dans le schéma d'action, la casse des pôles manuels donne l'importance de la quantité de mouvement.

\section{Expérience d'association d'étiquettes sur présentation de réalisations gestuelles}

Pour 20 UG nous analysons la validité de la catégorisation gestuelle fondée sur les principes de physiologie articulaire énoncés plus haut, à 
travers un test d'association d'étiquettes à des réalisations gestuelles ${ }^{8}$. Il s'agit de présenter des réalisations gestuelles sous forme vidéo respectant les schémas d'action, hors de tout contexte verbal, à raison de 2 séries de 10 gestes chacun. Ces réalisations impliquent toutes au moins le mouvement du segment substrat. Chaque geste numéroté est présenté 3 fois de suite à 41 sujets. Pour chaque geste présenté, les sujets doivent sélectionner une seule étiquette sur 10. L'ordre de présentation des gestes n'est pas aléatoire : ils sont répartis dans l'une des 2 séries des 10 étiquettes dès lors qu'ils sont jugés comme proches. 41 sujets $^{9}$ ont passé les deux séries de test.

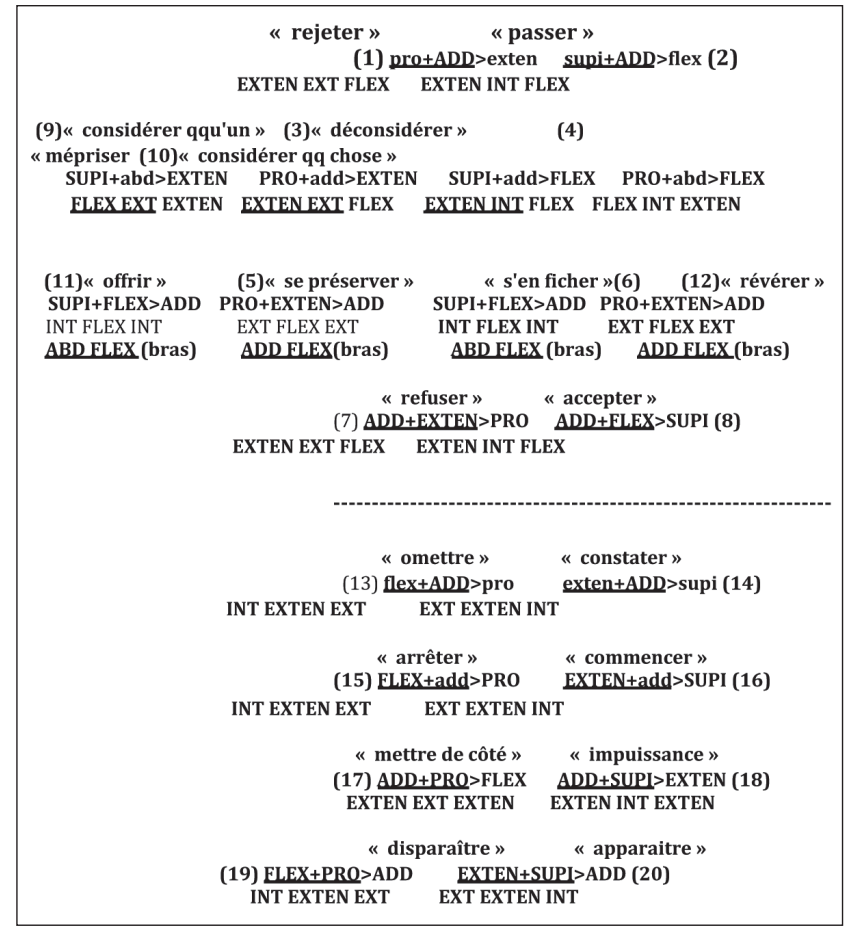

Tableau 1. - 20 Unités gestuelles avec les étiquettes et les schémas d'action.

8. Cette étude bénéficie du soutien de l'Agence nationale de la recherche (ANR) dans le cadre du projet CREAGEST.

9. 30 femmes et 11 hommes, étudiants en IUT dont la moyenne d'âge est de 21 ans et 6 mois. 
Nous testons ici la potentialité des gestes à être catégorisés de manière significative, d'une part, et, d'autre part, à l'être avec une étiquette attendue. Si c'est le cas, les gestes proposés sur une base de constitution purement physiologique révèleront alors que celle-ci est non seulement sémantique mais qu'elle organise aussi les sens en fonction d'une structuration qui repose sur les linéaments du corps.

La situation du test met en présence deux systèmes sémiotiques présentant des proximités respectives entre éléments. On peut dégager à priori deux types de proximité, celle formelle des gestes présentés et celle sémantique des étiquettes proposées.

La première s'apprécie d'abord à l'aune des centres d'organisation et des pôles des degrés de liberté en mouvement dans le schéma d'action ${ }^{10}$ : les schémas d'action les plus proches partagent les mêmes pôles, dans le même ordre (tels les gestes 1 et 3 , voir tableau 1, supra), puis les mêmes pôles dans un ordre différent au moins pour un segment (les gestes 1 et 7), ensuite, toujours les mêmes pôles mais cette fois l'ordre différent touche deux segments ( 1 et 5 , la main et l'avant-bras). Toujours pour les $20 \mathrm{UG}$, la proximité gestuelle diminue encore lorsqu'un des pôles du segment organisateur est inversé dans un autre schéma. Pour l'avant-bras (UG 3 et 4, 9 et 10, Rot.EXT vs Rot.INT), cette inversion entraine deux inversions polaires sur le segment support, c'està-dire la main (non générateur de l'UG). Lorsque la main constitue le segment substrat (UG 1, 2, 5, 6, 7, 8 et de 13 à 20), une seule inversion polaire entraine automatiquement un changement d'ordre sur le segment substrat (UG 1 et 13 par exemple). Lorsque les deux pôles de la flexion/extension et de la pronosupination de la main substrat sont inversés et pour une même quantité de mouvement (même ordre dans les schémas) alors on a un antonyme («rejeter»1 [pro+ADD>exten] vs «passer» 2 [supi+ADD $>$ flex], «refuser» 7 [ADD+EXTEN>PRO] $v s$

10. Avant même de pouvoir mesurer la proximité de schémas d'actions constitués, on doit considérer la filiation gestuelle, phénomène par lequel le substrat diffuse son mouvement sur le support et capture des zones d'identité sur les segments qui composent alors une part de l'enveloppe du schéma d'action. Pour un centre d'organisation à forte inertie comme l'épaule, le flux de propagation du mouvement - proximal distal - déborde les limites du membre supérieur. Il peut même s'inverser, une fois le bout des doigts atteint par le mouvement, et aller à rebours, selon un flux distal proximal. C'est le cas des gestes 17 et 18 dont les schémas d'action sont les excroissances anadromiques d'UG générées sur l'épaule. 
«accepter» 8 [ADD+FLEX $>$ SUPI]). Si en plus de cette double inversion l'ordre des degrés de liberté n'est pas le même, la distance s'accroit encore. L'antonymie ne constitue donc pas la distance gestuelle la plus importante.

À côté de cette distance gestuelle, les étiquettes présentent elles aussi une distance propre non homéomorphe à cette dernière. Par-delà la signification attachée à chaque étiquette, la polysémie associée (Victorri et Fuchs, 1996) amène pour le test une lecture catégorisante de ces étiquettes. Chacune apporte dans sa globalité même un ensemble de sens qui doivent servir de cibles privilégiées à partir d'un geste source. Chaque cible/étiquette, multiple, ne partage évidemment pas les mêmes frontières avec le geste auquel elle sera associée par les sujets. Il s'agit plutôt de vérifier que des UG dont la constitution repose sur des principes physiologiques fonctionnent néanmoins comme des entités sémantiques distinguables malgré la proximité de formes des gestes présentés, d'une part, et, malgré le réseau sémantique serré des étiquettes, d'autre part.

\section{Résultats}

L'inférence statistique permettant de déterminer la significativité des résultats est la loi binomiale (voir tableau 2). On considère qu'en deçà de $5 \%$ de chance que le hasard intervienne dans le choix d'étiquette fait par l'ensemble des sujets, on a bien un choix significatif des étiquettes de la part des sujets. L'inférence statistique utilisée ici mesure la probabilité d'intervention du hasard dans le choix des étiquettes: en dessous d'une probabilité de $5 \%$, le choix ne relève plus de l'aléatoire, il est fait de manière significative par les sujets.

18 réalisations gestuelles sur 20 sont étiquetées de manière significative par le mot attendu. Cela signifie que le modèle de structuration physiologique est validé, puisque $90 \%$ des UG fonctionnent comme des entités susceptibles d'être mises dans une catégorie sémantique unique. En outre, derrière cette catégorisation unique, les étiquettes proposées correspondent, pour $90 \%$ des cas aussi, aux étiquettes attendues. Les deux unités gestuelles qui sont reconnues de manière à peine significative (Probabilités 0,02; UG 15 [étiquette attendue : «arrêter»] et 19 [étiquette attendue : «disparaitre»]) sont néanmoins de préférence étiquetées «refuser» et «arrêter», étiquettes qui, par ailleurs, sont significativement attribuées aux UG 7 et 3, comme nous l'attendions. La seule différence entre les schémas d'actions des UG présentés et des étiquettes non attendues réside dans l'alternance flexion/extension 
manuelle pour l'une - les deux pôles des deux autres degrés de liberté restent identiques ${ }^{11}$ - et dans une proximité d'ordre pour l'autre ${ }^{12}$. Y compris pour les deux seules mauvaises attributions, on observe ainsi que la structuration physiologique est respectée.

\begin{tabular}{|c|c|c|c|}
\hline Test 1 & Prob. Hasard & Test 2 & Prob. Hasard \\
\hline apparaitre & $6,997 * 10^{-6}$ & impuissance & $1,116^{*} 10^{-9}$ \\
\hline disparaitre & 0,02 & mettre de côté & $3,31^{*} 10^{-26}$ \\
\hline s'en ficher & $3,885^{*} 10^{-31}$ & rejeter & $2,955^{*} 10^{-23}$ \\
\hline constater & $5,761 * 10^{-3}$ & passer & $1,773 * 10^{-12}$ \\
\hline refuser & $1,499 * 10^{-6}$ & se préserver & $7,202 * 10^{-35}$ \\
\hline offrir & $1,577 * 10^{-20}$ & omettre & $5,761 * 10^{-3}$ \\
\hline arrêter & 0,02 & commencer & $1,043^{*} 10^{-3}$ \\
\hline $\begin{array}{c}\text { considérer } \\
\text { quelque chose }\end{array}$ & $1,088^{*} 10^{-4}$ & mépriser & $3,593 * 10^{-4}$ \\
\hline déconsidérer & $1,701 * 10^{-13}$ & révérer & $1,436^{*} 10^{-10}$ \\
\hline accepter & $1,116^{*} 10^{-9}$ & $\begin{array}{c}\text { considérer } \\
\text { quelqu'un }\end{array}$ & $1,116^{*} 10^{-9}$ \\
\hline
\end{tabular}

Tableau 2. - Probabilités d'intervention du hasard pour les tests 1 et $2(P<0,05)$.

\section{Proximités}

Parmi les 18 UG reconnues, d'autres étiquettes ont pu être attribuées de manière significative quoique dans une moindre mesure que celle choisie de préférence. On ne peut s'attendre à ce que des gestes non répertoriés comme emblèmes ${ }^{13}$ soient alloués à une seule étiquette à l'exclusion d'autres, surtout lorsque ces dernières sont proches («considérer

11. Par ailleurs, les deux UG non reconnues ici l'ont été dans un autre test présentant à 66 sujets les UG du deuxième bloc uniquement (de 13 à 20) : pour UG $19, \mathrm{P}=1,79 * 10^{-6}$ et pour l'UG $15, \mathrm{P}=3,13^{*} 10^{-10}$.

12. La seule différence entre L'UG 19 «disparaitre» (FLEX+PRO>ADD) plutôt étiquetée «arrêter» et son UG (13) à laquelle cette étiquette renvoie (FLEX+add>PRO) réside dans l'ordre des pôles de la composante manuelle.

13. En dehors de l'UG 6 («s'en ficher») répertoriée dans Geneviève Calbris (1990, p. 4, ill. 1.4) et de l'UG 7 («refuser») répertoriée (ibid., p. 74), les autres UG ne figurent pas comme telles dans les emblèmes retenus par cette auteure. 
quelqu'un» et «révérer», «omettre» et «mettre de côté» ou bien «mettre de côté» et «rejeter», «accepter» et «constater» par exemple). En outre, les plus fortes proximités gestuelles sont nombreuses parmi les UG des deux tests : 2 trios et 5 paires d'UG partagent les mêmes pôles manuels, pour lesquels seul l'ordre d'enchainement du mouvement varie (proximité d'ordre : voir par exemple UG 1, 5, 12 pour un trio et 16, 18 pour un duo). Les doubles étiquetages surviennent 12 fois dans le premier test et 6 fois avec les UG du deuxième test ${ }^{14}$. Pourtant ceux-ci ne concernent jamais les proximités d'ordre, soit les plus grandes. Ainsi, il n'y a pas de confusion dans la forme des gestes, fût-ce pour une simple différence d'ordre des composantes du mouvement : à grande proximité, parfaite distinction. En fait, outre cette quasi-identité gestuelle jamais confondue dans ces tests, trois autres facteurs de proximité sont à examiner ici.

1. Le premier concerne le type de degrés de liberté qui change à travers le double étiquetage. Il est déterminé en comparant le schéma d'action de l'UG source avec celui de l'étiquette attribuée à tort (UG cible). Ainsi, l'UG 14 «constater» bien étiquetée, est aussi étiquetée «offrir» (UG 11, cible). La différence de degrés de liberté entre les deux schémas d'action est polaire : l'UG source (14) contient une extension tandis que l'UG cible (11) présente une flexion. La confusion concerne donc ici la flexion/extension. Nous verrons que le type de degrés de liberté est concordant avec le changement sémantique.

2. Le deuxième type de proximité relève du nombre de pôles inversés entre l'UG source et celle(s), cible(s), pointée(s) par l'(es) étiquette(s) attribuée(s) de manière abusive. On peut avoir de 1 à 3 pôles inversés : l'exemple précédent présente une seule inversion, comme par exemple entre l'UG 10 (source) et l'UG 15 (cible), un pôle est inversé : l'abduction/adduction. Ce critère relève plutôt de la forme.

3. Le dernier type de proximité a trait à la position du pôle qui change dans la composante manuelle du schéma d'action entre les UG source et cible. Trois positions sont susceptibles de changer. Les deux premières relevant d'un mouvement volontaire, on

14. Par exemple dans le test 2 , l'UG «rejeter» bien reconnue $\left(2,955^{*} 10^{-23}\right)$ est également étiquetée «mépriser» à hauteur de $5,76^{*} 10^{-3}$ probabilité que le hasard intervienne dans ce choix. 
suppose qu'un véritable choix s'y opère. La troisième position, toujours induite, n'est modifiable qu'à la seule condition d'un changement d'ordre des deux premiers pôles en mouvement. Ce n'est jamais le cas ici ou bien par modification polaire d'au moins une des deux premières positions (voir supra entre l'UG source 19 et l'UG cible 15). Le statut de ce critère est difficilement qualifiable.

Quelles composantes en termes de pôles sont confondues? Voyons d'abord celle qui n'est jamais confondue : les schémas d'action visés par une étiquette cible jouant de la seule inversion polaire pronation/supination n'apparaissent jamais. De manière générale, sur 18 doubles étiquetages -12 du test 1 et 6 du test $2-, 8$ concernent la seule alternance polaire flexion/extension, 2 celle de l'abduction/adduction simple, zéro pour la pronation/supination - on vient de le voir - et enfin on a 6 doubles inversions (flex/exten et pro/supi, par exemple) ainsi que 2 cas d'inversion polaire pour les 3 degrés de liberté manuels. On a donc une forte représentation de la flexion/extension, comme si à lui tout seul le changement polaire de ce degré de liberté n'opérait pas de changement important dans la signification des gestes.

À l'opposé, la pronation/supination semble impliquée dans le changement sémantique, dans le double étiquetage des UG : son absence d'inversion polaire unique le prouve. Au demeurant rappelons que la pronosupination est le seul ddl à agréger autant de mouvement induit provenant de trois segments du membre supérieur, le rendant ainsi réceptacle potentiel de significations, et le seul de la main à ne pas présenter de transfert inertiel vers l'avant-bras ou le bras - marquant son absence de diffusion trans-segmentale de la signification. Ce dernier point est au contraire fortement assumé par l'abduction/adduction à cause de son amplitude limité $\left(50^{\circ}\right)$. L'abd/add fonctionne comme un rapide diffuseur trans-segmental des UG organisées sur la main. Couplé avec sa position dans le schéma d'action, il constitue un bon indice de distribution des centres d'organisation. En première position et en partie en deuxième, il marque une organisation manuelle des UG. Lorsqu'il est en deuxième position (pour une faible quantité) et en troisième position, la main en quelque sorte subit un flux proximal-distal de propagation du mouvement donné par des segments supérieurs à inertie plus grande : la main est alors non plus substrat mais support d'un geste. C'est le cas ici pour les UG 3, 4, 5, 6, 9, 10, 11 et 12 . Le rôle de l'abduction/adduction dans la signification des UG n'est pour l'instant guère plus éclairci. 
À propos du deuxième type de proximité - celui du nombre de pôles inversés - un gradient à priori décroissant existe d'une seule inversion polaire jusqu'à trois possibles entre deux schémas d'action (dans leur composante manuelle). Moins il existe de pôles inversés, plus les schémas d'action sont proches et plus on peut s'attendre à une confusion entre les gestes, marquée par un double étiquetage. Les faits appuient cette conception : sur 18 doubles étiquetages d'UG, environ $56 \%$ sont issues d'inversions polaires simples, $34 \%$ d'inversions polaires doubles, et à peu près $11 \%$ d'inversions polaires triples. L'étiquetage sémantique, y compris dans ces erreurs repose, sur un modèle gestuel prévisible, qualifiable par la physiologie articulaire.

Le troisième facteur de proximité (et en conséquence de distance), la position des inversions polaires dans le schéma d'action, semble entretenir un rapport étroit avec au moins l'un des deux autres facteurs de proximité, on vient de le voir avec l'abduction/adduction, marqueur des centres d'organisation. À regarder les UG dans le tableau 1, on voit nettement que pour le seul changement d'ordre des pôles en mouvement dans les schémas d'action sans inversion polaire, la proximité la plus grande opère, les étiquettes en font foi («rejeter» 1 , «déconsidérer» 3 , «refuser» 7 ou encore, «disparaitre»19, «arrêter» 15 , «omettre» 13 et «mettre de côté»17), quand bien même cette proximité ne donne lieu dans les tests à aucune confusion. Par ailleurs, l'ordre dans le schéma d'action et par conséquent la position dans le schéma dépend toujours de la quantité de mouvement d'un autre ddl en mouvement et dépend aussi de l'amplitude restant à parcourir jusqu'à la butée articulaire. En somme, la position dans un schéma d'action est déterminée par la quantité de mouvement des deux premiers ddl; on ne peut envisager l'une sans prendre en compte l'autre. En outre les ddl - y compris chacun des pôles - réagissant différemment à l'endroit du transfert possible de leur mouvement, dans la pratique chaque UG constitue un cas quasi particulier. Pourtant, en théorie, on doit pouvoir suivre jusqu'à l'enchainement des proximités des schémas gestuels et donc des UG.

Pour mieux déterminer les relations et les fonctions de l'ordre, de la position de l'inversion polaire ainsi que les relations entretenues avec le nombre d'inversions et le type de degrés de liberté touchés, les tests présentés ici ne sont évidemment pas suffisants. Il faut, d'une part, les étendre aux 20 autres UG qui ont à peine été évoquées ici et, d'autre part, multiplier les tests en variant les associations d'UG. 


\section{Conclusion}

Nous avons montré qu'une structuration physiologique d'unités gestuelles est susceptible de comporter une catégorisation étiquetable, à l'instar d'emblème, sans contexte verbal. Ces gestes pourtant très proches par leur forme (plus qu'aucun emblème) sont isolables; mieux, leur entour le plus immédiat n'est pas confondu. En outre, un réseau interdépendant de proximités compose des relations qui commencent à être explicitables et dont certains retentissements sémantiques émergent. En somme, on peut aborder la composition même de ces gestes à l'aide de critères internes qui interrogent tout autant la sémiose gestuelle que les spécificités du substrat. Montrer qu'une organisation aussi forte maintient et différencie tout à la fois des réalisations aussi ténues ne peut être sans conséquence sur les catégories en place dans le continuum gestuel de Kendon. À position identique dans le schéma d'action, l'alternance polaire de la pronosupination qui débouche sur des antonymes (UG 1 versus UG 2, ou bien 7 vs 8 ou encore 19 vs 20) rend caduc l'argument d'une augmentation de la convention pour les emblèmes puisque cette alternance est entièrement fondée sur un fait de nature (la physiologie articulaire). Au demeurant, il serait douteux qu'une organisation morpho-dynamique et sémantique valable pour un input gestuel de double mouvement volontaire soit remise en cause pour une entrée gestuelle mettant en mouvement un seul degré de liberté, autrement dit pour un geste dont la forme serait plus simple (comme un bâton?). Il reste à étendre l'étude de la structuration physiologique sur d'autres gabarits gestuels et à multiplier les tests d'assignation sémantique et de reconnaissance.

\section{RÉFÉRENCES BIBLIOGRAPHIQUES}

Boutet D. (2008) : «Une morphologie de la gestualité : structuration articulaire », Cahiers de linguistique analogique, $\mathrm{n}^{\circ}$ 5, p. 80-115.

Boutet D. et Cuxac C. (2008) : «Le signifiant gestuel : langue des signes et gestualité - Avant-Propos », Cahiers de linguistique analogique, $\mathrm{n}^{\circ} 5$, p. 2-15.

CAlbris G. (1990) : The Semiotics of French Gestures, Bloomington, Indiana University Press, $236 \mathrm{p}$.

Cheng P. L. (2006) : «Simulation of Codman's paradox reveals a general law of motion », Journal of Biomechanics, vol. 39, n 7, p. 1201-1207.

Kapandu I.A. (1997) : Physiologie articulaire. 1, Membre supérieur, Paris, Maloine, $295 \mathrm{p}$. 
Kendon A. (1988) : «How Gestures Can Become like Words», dans F. Poyatos (éd.), Cross-Cultural Perspective in Nonverbal Communication, Toronto, C. J. Hogrefe, p. 131-141.

Kendon A. (2004) : Gesture: visible action as utterance, Cambridge, Cambridge University Press, $400 \mathrm{p}$.

MCNeILl D. (1992) : Hand and mind: what gestures reveal about thought, Chicago, Londres, University of Chicago Press, 416 p.

McNeill D. (2000) : Language and gesture, Cambridge, Cambridge University Press, $409 \mathrm{p}$.

McNeILl D. (2005) : Gesture and thought, Chicago, Londres, University of Chicago Press, $318 \mathrm{p}$.

Politti J. C., Goroso G., Valentinuzzi M. E. et Bravo O. (1998) : «Codman's paradox of the arm rotations is not a paradox: mathematical validation », Medical Engineering \& Physics, vol. 20, n 4, p. 257-260.

Victorri B. et Fuchs C. (1996) : La polysémie : construction dynamique du sens, Paris, Hermès, 220 p. 